\title{
Diffractive supersymmetric particle production at the CERN LHC
}

\author{
M. Boonekamp* \\ Service de physique des particules, CEA/Saclay, 91191 Gif-sur-Yvette cedex, France \\ J. Cammin ${ }^{\dagger}$ \\ University of Rochester, New York, USA \\ S. Lavignac ${ }^{\ddagger}$ and R. Peschanski ${ }^{\S}$ \\ Service de physique théorique, CEA/Saclay, 91191 Gif-sur-Yvette cedex, France ${ }^{\mathrm{T}}$ \\ C. Royon" \\ Service de physique des particules, CEA/Saclay, 91191 Gif-sur-Yvette cedex, France, \\ and Fermilab, Batavia, Illinois, USA \\ (Received 25 October 2005; published 28 June 2006)
}

\begin{abstract}
We give detailed predictions for the diffractive associated production of SUSY Higgs bosons and top squarks at the LHC via exclusive double pomeron exchange mechanism. We study how the SUSY Higgs production cross section and the signal-over-background ratio are enhanced as a function of $\tan \beta$ in different regimes. The prospects are particularly promising in the antidecoupling regime, which we study in detail. We also give prospects for a precise measurement of the top squark mass using the threshold scan of central diffractive associated top squark events at the LHC.
\end{abstract}

DOI: 10.1103/PhysRevD.73.115011

PACS numbers: $14.80 . \mathrm{Cp}$

\section{INTRODUCTION}

The quest for supersymmetry (SUSY) is one of the major goals in high energy particle physics, and is an important task for the experiments at the upcoming LHC. The discovery of SUSY Higgs scalar(s) and other new SUSY particles, among which the superpartners of the top quark are good candidates, would be an important achievement. Standard production mechanisms based on QCD are now well explored, at least for the main channels. However, due to both the general interest of the problem and some specific features of the SUSY Higgs and top squark sectors, one should seek for alternative ways of SUSY production. In this paper we investigate the prospects for diffractive production of SUSY Higgs bosons and associated sparticles (stops) in the central region of the detectors.

Standard model Higgs boson production in double diffraction (denoted DPE, for double pomeron exchange) has already been studied in recent years [1-8]. Many approaches have been pursued, considering diffractive scattering, as in the Regge picture [1-3], final state soft color interactions [6], or fully perturbative exchange of gluon

\footnotetext{
*Electronic address: boon@hep.saclay.cea.fr

†lectronic address: cammin@fnal.gov

Electronic address: lavignac@spht.saclay.cea.fr

${ }^{\S}$ Electronic address: pesch@spht.saclay.cea.fr

"Electronic address: royon@hep.saclay.cea.fr

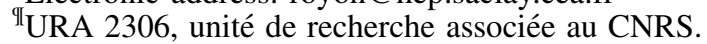

pairs [7]. We extend these studies to the SUSY Higgs and sparticle sector in the framework of the minimal supersymmetric standard model (MSSM) [9]. This subject has already been investigated in the literature $[10,11],{ }^{1}$ and we will, in particular, focus on central diffractive production of the lightest MSSM Higgs boson in the "antidecoupling" regime which has not yet been studied in the proposed framework. The "decoupling" and "intense-coupling" regimes have been studied in Ref. [13], where it is shown that diffractive Higgs boson production can help distinguishing between $h$ and $H$ in the intense-coupling regime.

\section{A. Central diffractive production of a heavy state}

One generally considers two types of DPE events for the production of a heavy state, namely, "exclusive" DPE [1-3], where the central heavy object is produced alone, separated from the outgoing hadrons by rapidity gaps:

$$
p p \rightarrow p+\text { heavy object }+p,
$$

and "inclusive" DPE [4-8], where the colliding Pomerons are resolved (very much like ordinary hadrons), accompanying the central object with Pomeron "remnants" $(X, Y)$ :

\footnotetext{
${ }^{1}$ A preliminary study of the diffractive SUSY Higgs in our framework, emphasizing the large enhancement factor at large $\tan \beta$, can be found in Ref. [12].
} 


$$
p p \rightarrow p+X+\text { heavy object }+Y+p .
$$

In general, exclusive production is considered most promising, because of a good expected signal-overbackground ratio (due to the large gaps with no or low hadronic activity specific of diffractive events) and because of the good missing mass resolution [14]. ${ }^{2}$ Obviously, hard diffractive cross sections are of higher order than standard hard nondiffractive ones, and this implies lower cross sections.

There are two objectives for this paper: First, to present a detailed calculation of the cross section for exclusive DPE events, and second, to elaborate on the advantage of using these events over other processes to search for new heavy objects and to determine their characteristics. Indeed, if the events are exclusive, i.e., if no other particles are produced in addition to the pair of heavy objects and the outgoing protons, the measurement of the scattered protons in roman pot detectors allows to access the Pomeron-Pomeron center-of-mass [14], and to study accurately the dynamics of the hard process. It is therefore possible to measure with great accuracy the properties of new particles, e.g. their mass. It is also possible to study the new couplings by measuring cross sections and angular distributions. As an example of this approach, we give a detailed description of the Higgs boson production cross section and the top squark mass measurement at production threshold. The method can easily be extended to other heavy objects.

These studies rely on the existence of exclusive events. At present, collider data from the Fermilab Tevatron give no evidence for the existence of exclusive production processes. Only upper bounds are given [15], dominated by the study of dijet events near the kinematic limit of the produced diffractive mass. It is particularly difficult to identify purely exclusive events experimentally, since the pomeron remnants are not detected, and the mass resolution measured in the D0 or CDF main detectors suffer large uncertainties due to the detector resolution. The evidence for exclusive events would be much clearer in the $\gamma$ channel since the measurement of the diphoton mass fraction does not suffer from these uncertainties. However, the production cross section of those events is expected to be small at the Tevatron and LHC data will be necessary to observe this process. Testing the present model with Tevatron data will thus be challenging and LHC data in the beginning of data taking will be of fundamental importance.

The theory of exclusive events is uncertain as well. Different models lead to cross sections at the LHC differing by orders of magnitude. Two approaches are commonly discussed in the literature [16], hereafter referred

\footnotetext{
${ }^{2}$ The missing mass can be computed very precisely using roman pot detectors, and is equal to twice the mass of the heavy object in the case of exclusive events.
}

to as the 'Pomeron-induced' [3,4] and 'proton-induced' [7] models ${ }^{3}$ for exclusive production. They share the common feature of satisfying the upper bounds for exclusive production at the Tevatron and they give similar predictions for a low-mass standard Higgs boson cross sections at the Tevatron. Apart from this, they come from different dynamics, since the proton-induced model is based on a semihard perturbative gluon mechanism, while the Pomeron-induced model is based on a soft, essentially nonperturbative, mechanism based on Pomeron exchange.

The Pomeron-induced model is an extension [3] to the purely exclusive processes of the original Bialas-Landshoff process for diffractive Higgs production [1] which was applied to heavy quark pairs in [2]. In our model, both inclusive and exclusive diagrams come from the same approach and are based on the Bialas-Landshoff model [1]. The model starts with the same soft Pomeron exchange diagrams (for ordinary dijet production the $g g \rightarrow g g$ diagrams are also included [17]) and correct the result by nonperturbative rapidity-gap survival factors [18,19]. The energy dependence is related to the rise of ordinary hadronic cross sections through features of the soft Pomeron [20].

An important issue of the Pomeron-induced model is that the colorless Pomeron exchange implies the diffractive phenomenon. Our model follows the Bialas-Landshoff model prescription, namely, we assume the existence of a direct coupling between the Pomeron and the heavy mass object which is produced (Higgs or jets for instance). Hence, by definition it takes into account the veto on gluon radiation from the production mechanism. In other words, since we consider a pomeron-induced model, there is no further need for a Sudhakov form factor to suppress radiation at the pomeron level. The counterpart is that it involves nonperturbative mechanisms which have to be modeled [1] using nonperturbative gluon propagators. Hence, in the Pomeron-induced framework, the normalization is not determined theoretically, since it is related to the unknown nonperturbative strong coupling constant $G^{2} / 4 \pi[1,2]$. However, the coupling constant value can be fixed phenomenologically by requiring consistency with the description of the inclusive dijet production within the same scheme, and is found [4] to be compatible with the value $G^{2} / 4 \pi=1$ chosen in [1,2]. For our study, we will use this value to evaluate the production cross sections.

The proton-induced model is based on the perturbative calculation related to the suppression of QCD radiation due to Sudakov form factors. Besides, soft radiation is forbid-

\footnotetext{
${ }^{3}$ We call 'proton-induced' the model of Ref. [7], since it has the unintegrated gluon distribution inside the proton as the main ingredient. This is contrasted with the 'Pomeron-induced' model $[3,4]$ where the main source are gluons in the Pomeron. The proton-induced and Pomeron-induced models are sometimes called, respectively, the "Durham model" and the "Saclay model" for exclusive production in the literature [16].
} 
den through a rapidity-gap survival formalism [18]. The same Sudakov form factors are responsible for a damping of large mass diffraction (for Higgs boson production, it is compensated by a rapid growth as a function of energy). In particular, one expects to get a negligible top squark pair exclusive cross section [21]. Indeed, in the proton-induced model approach, the normalization is estimated purely theoretically (except the lower bound constraints on dijet production) and leads to a top squark cross section too small to be observed at the LHC.

We will focus on exclusive production (1) and restrict ourselves to the original Bialas-Landshoff type of models $[1,2,4]$, with their extension to SUSY Higgs and stop production which we will develop in the next sections. There are large theoretical uncertainties of diffractive production which are related to the non trivial interplay between perturbative and nonperturbative contributions. We found it instructive to use the nonperturbative pomeroninduced model and compare it to the predictions for the proton-induced model. Indeed, it will be straightforward to extend these studies to other models. A recently developed Monte Carlo program, DPEMC [22], implements the models of $[1-3,5,7]$. Moreover, most of the plots are in terms of $s / b$ and enhancement factors, that are independent of the models.

Even though inclusive DPE (2) is a less promising search channel, it is still important to consider. In particular, it is interesting to evaluate the tail of the inclusive mass spectrum ("quasiexclusive" processes) since it constitutes a background to exclusive DPE. In addition, only inclusive DPE has actually been observed for high central masses [15]. Issues of inclusive DPE will be discussed in a future publication.

\section{B. The relevant SUSY spectrum: SUSY Higgs boson and top squarks}

Because of the limitation in the available total energy for production, diffractive production is favored for the production of SUSY particles in the lower range of their mass in the admissible set of model parameters. Hence, we will focus on this range for the SUSY Higgs and top squarks sector. The regions of the MSSM parameter space that favor a light Higgs boson and those that favor a light top squarks are not the same, so a specific study is required separately for Higgs bosons and top squarks, see Sec. III and IV.

It is well-known that the Higgs boson sector of the MSSM is richer than that of the standard model (SM). First, it contains five physical scalar degrees of freedom, instead of a single one: two $C P$-even neutral Higgs bosons $h$ and $H$, a pseudoscalar Higgs bosons $A$ and a charged Higgs boson pair $H^{ \pm}$. Secondly, the lightest MSSM Higgs boson $h$ may look very different from the SM Higgs boson. One can define (at least) three noteworthy regimes for the couplings of the neutral MSSM Higgs bosons $h, H$ and $A$ : (i) the decoupling regime, in which $h$ behaves like the SM Higgs boson [23,24];

(ii) the intense-coupling regime, in which the couplings of all three neutral Higgs bosons are very different from those of the SM Higgs boson [25];

(iii) the so-called antidecoupling regime [26], in which $H$ behaves like the SM Higgs boson, while $h$ has enhanced (resp. suppressed) couplings to downtype fermions (resp. up-type fermions and gauge bosons) [27].

It is also well-known that the MSSM Higgs boson sector contains at least one scalar $h$ with rather low mass (the other, $H$, being with larger but possibly accessible mass) which gives a particular interest for diffractive production as we will study in detail in this paper. Indeed, the small mass, the sometimes small rate and the experimentally difficult standard channels, e.g., the decay into $\gamma \gamma$, enhances the interest in alternative production modes and decays such as diffractive production. We will, in particular, focus on central diffractive production of the lightest MSSM Higgs boson in the antidecoupling regime (iii) which has not yet been intensively studied in the proposed framework. The decoupling and intense-coupling regimes have been studied in Ref. [13], where it is shown that diffractive Higgs boson production can help to distinguish between $h$ and $H$ in the intense-coupling regime.

The interest of MSSM Higgs boson production via exclusive diffractive production parallels a similar analysis for the standard model Higgs boson, with some distinctive features which enhance the specific production and branching modes.

The discovery of "sparticles" at the LHC would be the clearest and most exciting signal of new fundamental physics beyond the standard model. Among these, the scalar superpartners of the top quark are expected to be those with smallest mass among scalars in a large portion of the MSSM parameter space. Indeed, various supersymmetric scenarios can accommodate a light top squark consistent with the experimental bounds on other sparticle masses and with measurements of observables that could be affected by large supersymmetric contributions, such as the anomalous magnetic moment of the muon or the branching ratio of the flavor-violating decay $b \rightarrow s \gamma$. Minimal supergravity [28] (mSUGRA) scenarios with a light top squark typically require a low gaugino mass parameter $m_{1 / 2}$ and a large $A$-term parameter $A_{0}$. The need for a small $m_{1 / 2}$ is due to the fact that the renormalization group equations for the soft-supersymmetrybreaking squark masses $M_{Q}^{2}$ and $M_{R}^{2}$ receive a large contribution from gluinos: the larger $m_{1 / 2}$, the higher the weak-scale values of $M_{Q_{3}}^{2}$ and $M_{U_{3}}^{2}$. As an example, the Snowmass Point 5 (SPS 5), defined by the following values of the mSUGRA parameters: $m_{0}=150 \mathrm{GeV}, m_{1 / 2}=$ $300 \mathrm{GeV}, A_{0}=-1000 \mathrm{GeV}, \tan \beta=5$ and $\operatorname{sign}(\mu)=$ + , yields the following top squark and sbottom spectrum 
[29], as generated by the program SUSYGEN 3.00/27 [30]:

$$
\begin{array}{ll}
m_{\tilde{t}_{1}}=210 \mathrm{GeV}, & m_{\tilde{t}_{2}}=632 \mathrm{GeV}, \\
m_{\tilde{b}_{1}}=561 \mathrm{GeV}, & m_{\tilde{b}_{2}}=654 \mathrm{GeV} .
\end{array}
$$

For comparison, a "typical" mSUGRA scenario with vanishing $A_{0}$, the "post-WMAP benchmark scenario" B', defined by $m_{0}=60 \mathrm{GeV}, m_{1 / 2}=250 \mathrm{GeV}, A_{0}=0$, $\tan \beta=10$ and $\operatorname{sign}(\mu)=+$, yields [31]:

$$
\begin{array}{ll}
m_{\tilde{t}_{1}}=393 \mathrm{GeV}, & m_{\tilde{t}_{2}}=573 \mathrm{GeV}, \\
m_{\tilde{b}_{1}}=502 \mathrm{GeV}, & m_{\tilde{b}_{2}}=528 \mathrm{GeV} .
\end{array}
$$

Light top squarks and bottom squarks ${ }^{4}$ can also arise from nonminimal SUGRA models, e.g. from scenarios with an inverted mass hierarchy in the squark sector [32]. Assuming $M_{\Phi_{3}}^{2} \ll M_{\Phi_{1,2}}^{2}(\Phi=Q, U, D)$ at the GUT scale and small gaugino masses, one ends up with very low third generation squark masses at the weak scale due to strong 2loop renormalisation group effects proportional to $\alpha_{S}^{2} \operatorname{Tr}\left(2 M_{Q}^{2}+M_{U}^{2}+M_{D}^{2}\right)$ [33]. On the contrary, the first two squark generations remain heavy. The top squark and sbottom squared masses can even be driven negative if the GUT-scale hierarchy $M_{\Phi_{3}}^{2} \ll M_{\Phi_{1,2}}^{2}$ is too pronounced, or if the gluino mass, whose contribution to the running of the squark masses tends to compensate for the two-loop gauge contribution, is too small.
The paper is organized in the following way. In the next Sec. II, we introduce the concept of central diffractive production of SUSY Higgs bosons and top squarks; in II A, the formalism of exclusive production and in II B the experimental context are presented. In Sec. III, we focus on the MSSM Higgs boson sector; in III A, theoretical aspects of the Higgs boson spectrum and in III B, the predictions for the LHC are displayed. In Sec. IV, the case for top squark, and eventually bottom squark production is discussed; in IVA, the theoretical framework, in IV B, the predicted cross sections and missing mass distribution and in IV C, the top squark mass measurement by a threshold scan are given. The paper ends by a conclusion and outlook.

\section{DIFFRACTIVE PRODUCTION OF SUSY HIGGS BOSON AND TOP SQUARKS}

\section{A. Exclusive central diffractive production}

In this section we introduce the model $[1,2,4]$ that is being used to describe exclusive MSSM Higgs bosons and top squark pair production in double diffractive production. In [1,2], the diffractive mechanism is based on twogluon exchange between the two incoming protons. The soft pomeron is seen as a pair of gluons coupled nonperturbatively to the proton. One of the gluons is then coupled perturbatively to the hard process, either the SUSY Higgs bosons, or the $\tilde{t} \overline{\tilde{t}}$ pair, while the other one plays the rôle of a soft screening of color, allowing for diffraction to occur. The corresponding cross sections for Higgs bosons and $\tilde{t} \overline{\tilde{t}}$ production read:

$$
\begin{aligned}
& d \sigma_{h}^{\mathrm{exc}}(s)=C_{h}\left(\frac{s}{M_{h}^{2}}\right)^{2 \epsilon} \delta\left(\xi_{1} \xi_{2}-\frac{M_{h}^{2}}{s}\right) \prod_{i=1,2}\left\{d^{2} v_{i} \frac{d \xi_{i}}{1-\xi_{i}} \xi_{i}^{2 \alpha^{\prime} v_{i}^{2}} \exp \left(-2 \lambda_{h} v_{i}^{2}\right)\right\} \sigma(g g \rightarrow h) \\
& d \sigma_{\tilde{t} \tilde{t}}^{\operatorname{exc}}(s)=C_{\tilde{t} \tilde{\tilde{t}}}\left(\frac{s}{M_{\tilde{t} \tilde{t}}}\right)^{2 \epsilon} \delta^{(2)}\left(\sum_{i=1,2}\left(v_{i}+k_{i}\right)\right) \prod_{i=1,2}\left\{d^{2} v_{i} d^{2} k_{i} d \xi_{i} d \eta_{i} \xi_{i}^{2 \alpha^{\prime} v_{i}^{2}} \exp \left(-2 \lambda_{\tilde{t} \tilde{t}} v_{i}^{2}\right)\right\} \sigma(g g \rightarrow \tilde{t} \tilde{\bar{t}})
\end{aligned}
$$

where, in both equations, the variables $v_{i}$ and $\xi_{i}$ denote the transverse momenta and fractional momentum losses of the outgoing protons. In the second equation, $k_{i}$ and $\eta_{i}$ are the squark transverse momenta and rapidities. $\sigma(g g \rightarrow H)$, $\sigma(g g \rightarrow \tilde{t} \tilde{\bar{t}})$ are the hard production cross sections which are given later on. The model normalization constants $C_{h}$, $C_{\tilde{t} \tilde{t}}$ are fixed from the fit to dijet diffractive production, and are given in Ref. [1,2,22].

In the model, the soft pomeron trajectory is taken from the standard Donnachie-Landshoff parametrization [20], namely $\alpha(t)=1+\epsilon+\alpha^{\prime} t$, with $\epsilon \approx 0.08$ and $\alpha^{\prime} \approx$

\footnotetext{
${ }^{4}$ In the following, we consider only top squarks. But the study remains unchanged for squarks (i.e., bottom squarks) if their masses are sufficiently low.
}

$0.25 \mathrm{GeV}^{-2} . \lambda_{h}, \lambda_{\tilde{t} \tilde{t}}$ are kept as in the original paper $[1,2]$ for the SM Higgs boson and $q \bar{q}$ pairs. ${ }^{5}$ Note again that, in this model, the strong (nonperturbative) coupling constant is fixed to a reference value $G^{2} / 4 \pi=1$.

In order to select exclusive diffractive states, one needs to take into account the corrections from soft hadronic scattering. Indeed, the soft scattering between incident particles tends to mask the genuine hard diffractive interactions at hadronic colliders. The formulation of this correction $[18,19]$ leads to an overall correction factor of $3 \%$

\footnotetext{
${ }^{5}$ The expression of $C_{h}, C_{t \bar{t}}$ are explicitly given in formulas (2.13) and (2.14) of Ref. [1] and formulas (4) and (15) from Ref. [2], and included in DPEMC [22].
} 
which is. the commonly used correction factor $[4,7]$ for the QCD exclusive diffractive processes at the LHC.

\section{B. Experimental context}

The DPEMC [22] Monte Carlo program provides an implementation of the Higgs boson, top squark and bottom squark pair production described above in both exclusive and inclusive double pomeron exchange modes. It uses HERWIG [34] as a cross section library of hard QCD processes and when required, convolutes them with the relevant pomeron fluxes and parton densities. The survival probabilities discussed in the previous section $(0.03$ for double pomeron exchange processes) have been introduced at the generator level. The cross sections at the generator level are given in the next section after this effect is taken into account.

A possible experimental setup for forward proton detection is described in detail in [4,35]. We will only describe its main features here and discuss its relevance for the Higgs boson and top squark or bottom squark mass measurements.

In exclusive DPE or QED processes at the LHC, the mass of the central heavy object can be reconstructed using the roman pot detectors and tagging both protons in the final state. It is given by $M^{2}=\xi_{1} \xi_{2} s$, where $\xi_{i}$ are the proton fractional momentum losses, and $s$ is the total center-of-mass energy squared.

In the following, we assume the existence of two detector stations, located at approximately $210 \mathrm{~m}$ and $420 \mathrm{~m}$ [35] from the interaction point. The $\xi$ acceptance and resolution have been derived for each device using a complete simulation of the LHC beam parameters [35]. The combined $\xi$ acceptance is close to $\sim 60 \%$ at low masses of about $100 \mathrm{GeV}$, and $90 \%$ at higher masses starting at about $220 \mathrm{GeV}$ for $\xi$ ranging from 0.002 to 0.1 . In particular, this means that the low-mass objects (Higgs bosons or top squarks) are mainly detected in the $420 \mathrm{~m}$ pots whereas the heavier ones in the closer pots at $210 \mathrm{~m}^{6}$

Our analysis does not assume any particular value for the $\xi$ resolution. We will discuss in Secs. III B and IV C how the resolution on the Higgs boson or the top squark quark masses depend on the detector resolutions, or in other words, the missing mass resolution.

\section{SUSY HIGGS BOSON PRODUCTION}

\section{A. Theoretical aspects}

Let us briefly recall the properties of the lightest MSSM Higgs boson $h$ (for recent reviews, see Refs. [26,36]). As is well-known, $h$ is constrained to be lighter than the $Z$ boson at tree level. Once radiative corrections are taken into account [37-39], the upper limit on its mass becomes $m_{h} \lesssim 135 \mathrm{GeV}$. The actual value of $m_{h}$ depends on several MSSM parameters: two parameters that are sufficient to describe the Higgs boson sector at tree-level, generally chosen to be $m_{A}$ and $\tan \beta$, the ratio of the vacuum expectation values of the two Higgs boson doublets of the MSSM; and additional parameters that control the size of the radiative corrections. These are the top squark and bottom squark soft-supersymmetry-breaking masses, assumed in this paper to be degenerate and denoted by $M_{\text {SUSY }}$, the top squark and bottom squark triscalar couplings ( $A$-terms) $A_{t}$ and $A_{b}$, and the supersymmetric Higgs boson mass parameter $\mu$. The dependence of the lightest Higgs boson mass on these parameters can be roughly described as follows: $m_{h}$ increases with $m_{A}$ and $\tan \beta$, as well as with the common third generation squark mass $M_{\text {SUSY }}$. Its value also depends strongly on the top squark mixing parameter $X_{t} \equiv A_{t}-\mu \cot \beta$ : starting from the "minimal mixing" $X_{t}=0$, it increases with $X_{t}$ and reaches a maximum for $X_{t} \approx \sqrt{6} M_{S}$, where $M_{S}^{2} \equiv\left(m_{\tilde{t}_{1}}^{2}+m_{\tilde{t}_{2}}^{2}\right) / 2$ is the average of the two top squark squared masses $\left(M_{S} \simeq\right.$ $M_{\text {SUSY }}$ in the limit $\left.M_{\text {SUSY }} \gg m_{t}\right)$. This is illustrated by the following approximate formula for the one-loop upper bound on the lightest Higgs boson mass, valid in the decoupling limit $m_{A} \gg m_{Z}$ and for $m_{t} X_{t} \ll M_{S}^{2}[36,40]$ :

$m_{h}^{2} \leq m_{Z}^{2} \cos ^{2} 2 \beta+\frac{3 g^{2} m_{t}^{4}}{8 \pi^{2} m_{W}^{2}}\left[\ln \left(\frac{M_{S}^{2}}{m_{t}^{2}}\right)+\frac{X_{t}^{2}}{M_{S}^{2}}\left(1-\frac{X_{t}^{2}}{12 M_{S}^{2}}\right)\right]$.

In the minimal mixing case, $m_{h}$ can reach an upper limit of about $120 \mathrm{GeV}$ for $M_{\mathrm{SUSY}} \lesssim 1 \mathrm{TeV}$, while it can reach about $135 \mathrm{GeV}$ in the maximal mixing case [36].

The couplings of $h$ can significantly depart from those of the SM Higgs boson. In particular, its tree-level couplings to down-type and up-type fermions (normalized to the SM Higgs boson couplings) are given by:

$$
g_{h f f}= \begin{cases}\sin (\beta-\alpha)+\cot \beta \cos (\beta-\alpha) & (f=\text { up-type fermion }) \\ \sin (\beta-\alpha)-\tan \beta \cos (\beta-\alpha) & (f=\text { down-type fermion })\end{cases}
$$

\footnotetext{
${ }^{6}$ Information from the $220 \mathrm{~m}$ pots can be included into the first level of the trigger. However, the information from the pots located at $420 \mathrm{~m}$ come too late to reach the first level trigger, and can only be used in the second level trigger. Therefore, one must use either asymmetric events (one tag at $220 \mathrm{~m}$ and another one at $420 \mathrm{~m}$, where the trigger is based on the information from the $220 \mathrm{~m}$ pot) or directly a trigger from the main ATLAS and CMS detector. The latter option is challenging and under study in both collaborations.
} 
where $\alpha$ is the angle that diagonalizes the squared mass matrix of the $C P$-even Higgs boson and defines the physical $C P$-even states $h$ and $H$. As for the couplings to the gauge bosons, $h Z Z$ and $h W W$, they are suppressed by a factor $\sin (\beta-\alpha)$ relative to their SM values. In the decoupling regime $m_{A} \gg m_{Z}[23,24]$, in which $A, H$ and $H^{ \pm}$are all much heavier than $h,|\cos (\beta-\alpha)| \leq \mathcal{O}\left(m_{Z}^{2} / m_{A}^{2}\right) \ll 1$ and therefore the couplings of the lightest MSSM Higgs boson $h$ approach those of the SM Higgs boson (in particular, $g_{h f f} \simeq 1$ ). On the contrary, if $m_{A} \sim m_{Z}$ (or more precisely $m_{A}<m_{h}^{\max }$, where $m_{h}^{\max }$ is the maximal value $m_{h}$ can reach for fixed values of the squark parameters), $|\cos (\beta-\alpha)| \sim 1$ and therefore $h$ has significantly different couplings from those of the SM Higgs boson. In particular, at large $\tan \beta$, in the antidecoupling regime, its couplings to down-type fermions are strongly enhanced $\left(\left|g_{h b b}\right| \simeq\left|g_{h \tau \tau}\right| \simeq \tan \beta \gg 1\right)$, while its couplings to uptype fermions and gauge bosons are suppressed $\left(\left|g_{h t t}\right| \sim\right.$ $\cot \beta \ll 1$ and $g_{h W W}=g_{h Z Z}=\sin (\beta-\alpha) \ll 1$, in units of the SM Higgs boson couplings). As we shall see below, this enhances the production cross section of the lightest Higgs boson via gluon fusion, while the associated production with gauge bosons, $q \bar{q} \rightarrow Z h / W h$, is suppressed. Also the partial decay width of $h$ into $b \bar{b}\left(\tau^{+} \tau^{-}\right)$, which is proportional to $g_{h b b}^{2}\left(g_{h \tau \tau}^{2}\right)$, is enhanced. By contrast, the decay $h \rightarrow \gamma \gamma$, which in the decoupling regime is dominated by the $W$ boson loop, does not benefit from such an enhancement at large $\tan \beta$ (the subdominant bottom quark loop is enhanced, but the dominant $W$ boson loop is suppressed). This decay has therefore a suppressed branching ratio in the antidecoupling regime. We close this short review of the antidecoupling regime by noting that the heavier $C P$-even Higgs boson $H$, in contrast to $h$, has SM-like couplings, but is much heavier than $h$ and $A$. Finally, another regime of interest is the "intensecoupling" regime [25], which occurs when $m_{A} \sim m_{h}^{\max }$ and $\tan \beta$ is large. In this regime, all three neutral Higgs bosons are very close in mass, $m_{h} \approx m_{A} \approx m_{H}$, and have enhanced (suppressed) couplings to down-type fermions (down-type fermions and gauge bosons - the couplings $A W W$ and $A Z Z$ are forbidden by $C P$ invariance), so that it may be difficult to distinguish among them at the LHC.

Let us now discuss the production of the lightest MSSM Higgs boson via gluon fusion [41-48] (see Appendix A for the relevant formulas). In the SM, top quark loops give the main contribution to the cross section, and bottom loops give a smaller contribution. In the MSSM, the contribution of the bottom loops can become very large at large $\tan \beta$ (in the regime where the $h b b$ couplings, Eq. (A5), are enhanced) while the top quark loops are suppressed, resulting in an enhancement of the gluon fusion cross section. In addition, top and bottom squark loops contribute. However, top squark loops significantly affect the cross section only in the case of a light top squark, $m_{\tilde{t}_{1}} \lesssim$ (200-400) GeV. In the decoupling regime, their effects are particularly spectacular in the presence of a large top squark mixing, in which case they interfere destructively with the top quark contribution [49]. For bottom squark loops to be sizable, a large value of $\tan \beta$ is also needed, as well as a large value of $|\mu|$ in the decoupling regime.

In the regime we are interested in, which is characterized by a large value of $\tan \beta$ and a suppressed value of $\sin (\beta-$ $\alpha$ ), there is no enhancement of the $h \tilde{t} \tilde{t}$ couplings at large top squark mixing, contrarily to the situation in the decoupling regime. However, the $h \tilde{b} \tilde{b}$ couplings are enhanced at large mixing; but this is compensated by the fact that the bottom squark masses are generally larger than the lightest stop mass $m_{\tilde{t}_{1}}$ in typical MSSM scenarios. Therefore, we neglect the squark loops in the following discussion, although they are included in our numerical results. Neglecting as well the terms suppressed by $\cot \beta$, we then find the following enhancement factor for the MSSM cross section with respect to the SM cross section (the QCD corrections to the leading order cross sections are expected to reduce this ratio [26] by some 30\% at large $\tan \beta)$ :

$$
\begin{aligned}
\frac{\sigma_{\mathrm{MSSM}}(g g \rightarrow h)}{\sigma_{\mathrm{SM}}(g g \rightarrow h)} \approx & \mid \sin (\beta-\alpha)-\tan \beta \cos (\beta-\alpha) \\
& \times\left.\frac{A_{b}^{h}\left(\tau_{b}\right)}{A_{t}^{h}\left(\tau_{t}\right)+A_{b}^{h}\left(\tau_{b}\right)}\right|^{2},
\end{aligned}
$$

where the loop functions $A_{t}^{h}(\tau)$ and $A_{b}^{h}(\tau)$ are defined in Eq. (A2), and $\tau_{t(b)} \equiv m_{h}^{2} / 4 m_{t(b)}^{2}$. We therefore expect a large enhancement factor at large values of $\tan \beta$ in the regime where $m_{A}<m_{h}^{\max }$. This can indeed be seen in Fig. 1 (upper plot), where $\sigma_{\mathrm{MSSM}}(g g \rightarrow h) / \sigma_{\mathrm{SM}}(g g \rightarrow h)$ is shown as a function of $m_{h}$ for $\tan \beta=30$ and various values of the squark parameters. In the maximal mixing case, $m_{h}^{\max }$ is large and the antidecoupling condition $m_{A}<$ $m_{h}^{\max }$ is satisfied over the range $90 \mathrm{GeV} \leq m_{h} \leq 120 \mathrm{GeV}$ (remember that in this regime $\left.m_{h} \approx m_{A}\right)$, hence $\cos ^{2}(\beta-$ $\alpha$ ) remains very close to 1 , and the curve essentially reflects the dependence of the loop functions $A_{t}^{h}\left(\tau_{t}\right)$ and $A_{b}^{h}\left(\tau_{b}\right)$ on $m_{h}$. In the minimal mixing case, $m_{h}^{\max }$ is smaller, especially for $M_{\mathrm{SUSY}}=500 \mathrm{GeV}$ (namely $m_{h}^{\max } \approx$ $114 \mathrm{GeV}$ for $M_{\mathrm{SuSY}}=1 \mathrm{TeV}$, and $m_{h}^{\max } \approx 107 \mathrm{GeV}$ for $M_{\text {SUSY }}=500 \mathrm{GeV}$ ), so that one leaves the antidecoupling regime for much lower values of $m_{h}$ than in the maximal mixing case. This explains why the enhancement factor strongly decreases when $m_{h}$ approaches $m_{h}^{\max }$, and finally reaches the decoupling regime value $\sigma_{\mathrm{MSSM}}(g g \rightarrow$ $h) / \sigma_{\mathrm{SM}}(g g \rightarrow h)=1$ for $m_{h}=m_{h}^{\max }$ (up to squark loop effects, which remain small for the squark parameters considered here). Figure 1 (lower plot) shows the dependence of the enhancement factor on $\tan \beta$ for $m_{h}=$ $100 \mathrm{GeV}$. For this value of $m_{h}$, the condition $m_{A}<m_{h}^{\max }$ is satisfied for all four sets of the squark parameters considered. For moderate values of $\tan \beta(\tan \beta<5$ is ex- 

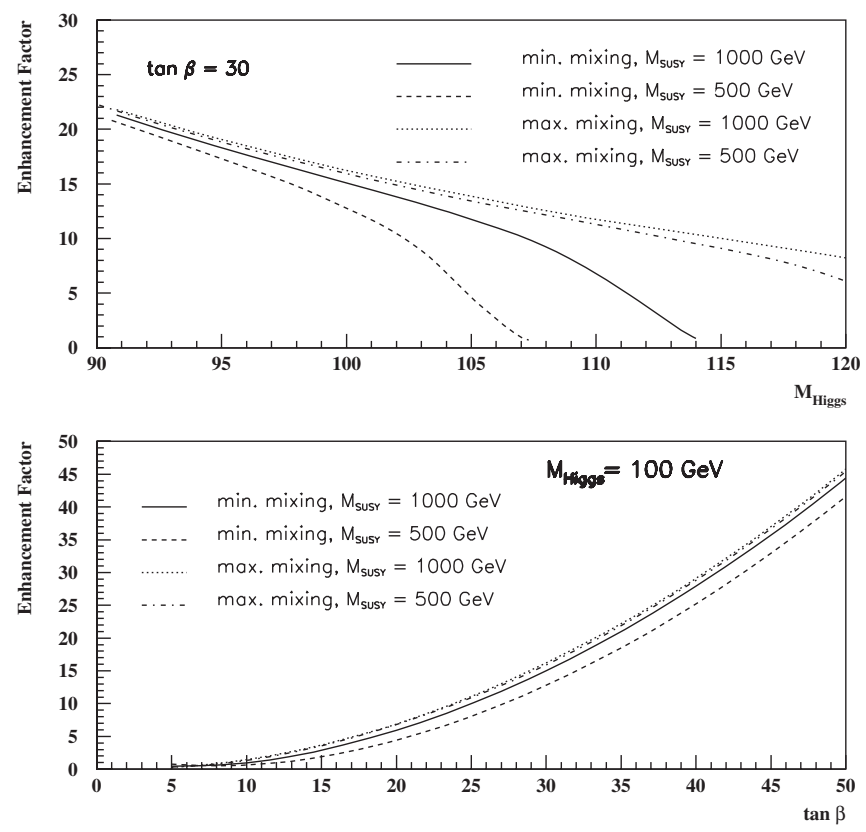

FIG. 1. Enhancement factor for the diffractive Higgs boson production cross section. Upper plot: enhancement factor as a function of the Higgs boson mass for a value of $\tan \beta=30$, and different mixing scenarii and SUSY masses (full line: minimal mixing, $M_{\mathrm{SUSY}}=1000 \mathrm{GeV}$; dashed line: minimal mixing, $M_{\mathrm{SUSY}}=500 \mathrm{GeV}$; dotted line: maximal mixing, $M_{\mathrm{SUSY}}=$ $1000 \mathrm{GeV}$; dashed dotted line: maximal mixing, $M_{\mathrm{SUSY}}=$ $500 \mathrm{GeV}$ ). Lower plot: similar study as a function of $\tan \beta$ for a Higgs boson mass of $100 \mathrm{GeV}$. The MSSM Higgs boson spectrum has been obtained using the program FeynHiggs [65], with $\mu=200 \mathrm{GeV}, \quad M_{3}=800 \mathrm{GeV}, \quad M_{2}=200 \mathrm{GeV}$ and $M_{1}=\left(\alpha_{1} / \alpha_{2}\right) M_{2}$.

cluded experimentally for $m_{h}=100 \mathrm{GeV}$ ), the antidecoupling regime is not yet reached, i.e., $\cos ^{2}(\beta-\alpha)$ is large but not maximal $\left(\cos ^{2}(\beta-\alpha) \sim 1\right)$. For larger values of $\tan \beta$, e.g., $\tan \beta \geq 20$, the antidecoupling regime is reached and the enhancement factor grows with $\tan ^{2} \beta$, as expected.

\section{B. SUSY Higgs boson production at the LHC}

In this section, we address the MSSM Higgs boson production for masses below $120 \mathrm{GeV}$, when the Higgs boson decays into $b \bar{b}$, the least favorable case at the LHC. As mentioned in the previous section, Fig. 1 shows the cross section enhancement factor for SUSY Higgs boson production with respect to the standard model case at generator level. In the upper plot of Fig. 1, the full and dashed lines show the results for the minimal mixing scenario for two common values of the mass, $M_{\mathrm{SUSY}}$, of third generation MSSM squarks (1000 and $500 \mathrm{GeV}$ ). They lead to typical masses of the top squark and bottom squarks of 1010 or $520 \mathrm{GeV}$, respectively. The cross section was computed using bottom, top, top squark and bottom squark loops, while the effect of top squark and bottom squark loops is less than one per mil. The enhancement factor can go up to a factor 20 compared to the standard model case, but is very dependent on the mass of the Higgs boson. In the maximal smearing scenario (dotted and dashed dotted curves in Fig. 1), the enhancement factor is found to be similar to that at low Higgs boson masses, but remains important at higher masses.

The bottom plot of Fig. 1 displays the dependence as a function of $\tan \beta$ for a Higgs boson mass of $100 \mathrm{GeV}$ and for the same scenarios as before. The enhancement factor for the Higgs boson production cross section can reach a factor of up to 45 for a value of $\tan \beta$ of 50 . For this particular value of the mass of the Higgs boson, the model dependence is not very large.

It is important to note that searches will benefit directly from the increase of the cross section since they will be looking for Higgs bosons decaying into $b \bar{b}$ in the main detector, and the branching ratio of $h \rightarrow b \bar{b}$ is quite stable as a function of the MSSM parameters in this region of phase space. ${ }^{7}$ Thus, the search for diffractive production of MSSM Higgs bosons is the only one benefiting fully from the increase of the cross section at high values of $\tan \beta$.

In the following, we perform a detailed study of signalover-background ratio in the case of a Higgs boson mass of $120 \mathrm{GeV}$. We chose this particular mass since in most of the MSSM parameter space, the Higgs boson mass is below this value, and this mass leads to the least favorable scenario (the lowest cross section and signal-over-background ratios) with respect to lower masses. In Fig. 2, we give the signal-over-background ratio for standard model and MSSM Higgs boson production for a mass of the Higgs boson of $120 \mathrm{GeV}$ and for different values of $\tan \beta$, as a function of the roman pot mass resolution (corresponding to the Higgs boson mass resolution) for a luminosity of $100 \mathrm{fb}^{-1}$. This study was performed after a fast simulation of the CMS detector [50] (the ATLAS detector simulation is expected to produce very similar results) and experimental cuts described in the following paragraph.

First of all, we require both final state protons to be detected in the roman pot detectors, and we take into account the acceptance of these detectors as it is discussed in Sec. I. The cuts applied in the analysis are detailed in Ref. [3]. The basic idea is to require two high $p_{T} b$-jets with $p_{T 1}>45 \mathrm{GeV}, p_{T 2}>30 \mathrm{GeV}$, originating from the decay of the Higgs boson into $b \bar{b}$ at low masses. The difference in azimuth between the two jets should be $170<\Delta \Phi<190 \mathrm{deg}$, asking the jets to be back-toback. Both jets are required to be central, $|\eta|<2.5$, $b$-tagged, with the difference in rapidity of both jets satisfying $|\Delta \eta|<0.8$. A cut is applied on the ratio of the dijet mass to the total mass of all jets measured in the calorim-

\footnotetext{
${ }^{7}$ This is not the case when one looks into nondiffractive MSSM Higgs bosons decaying into $\gamma \gamma$ which is strongly suppressed at high $\tan \beta$, see Sec. III A.
} 


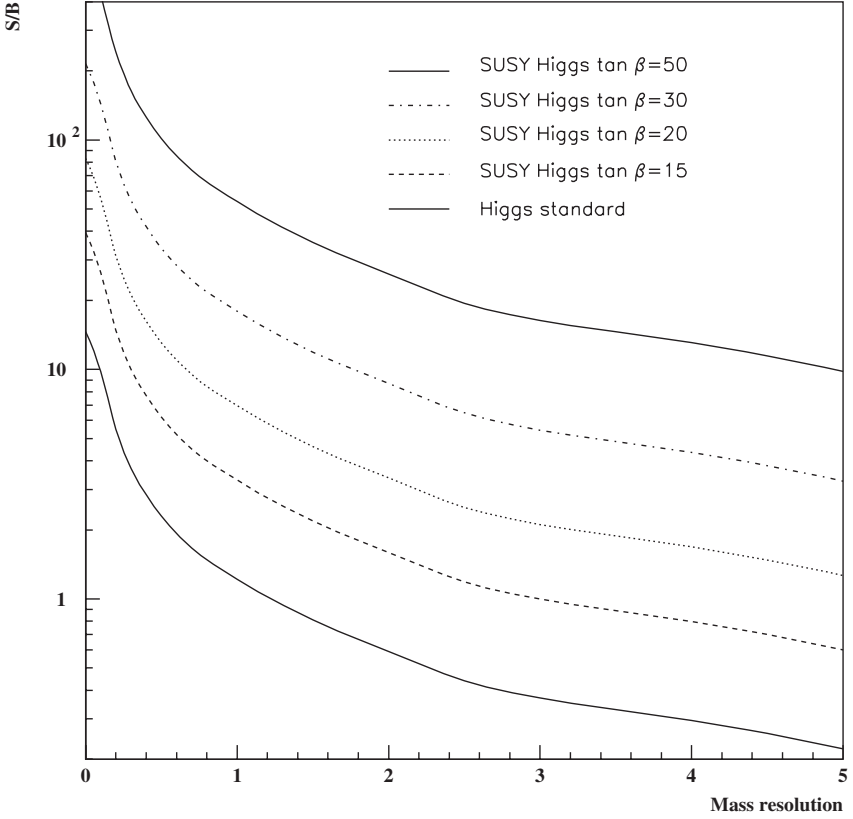

FIG. 2. Signal-over-background as a function of roman pot mass resolution for a Higgs boson mass of $120 \mathrm{GeV}$ and for different values of $\tan \beta$. From bottom to top: full line: standard model Higgs boson, dashed line: SUSY Higgs boson with $\tan \beta=15$, dotted line: $\tan \beta=20$, dashed dotted line: $\tan \beta=$ 30 , full line: $\tan \beta=50$.

eters, $M_{J J} / M_{\text {all }}>0.75$. The ratio of the dijet mass to the missing mass should fulfil $M_{J J} /\left(\xi_{1} \xi_{2} s\right)^{1 / 2}>0.8$.

The case for the standard model Higgs boson was already given in [3], and we follow the same approach concerning the background and signal studies. To compute the signal-over-background ratios, both signals and backgrounds dominated by exclusive $b \bar{b}$ production have been integrated over a $2 \mathrm{GeV}$ mass window. After cuts, the typical number of events expected for the signal of a $120 \mathrm{GeV}$ Higgs boson and for a luminosity of $100 \mathrm{fb}^{-1}$ is $27.1,73.2,154,398$ and 1198 for standard model and MSSM ( $\tan \beta=15,20,30$ and 50) Higgs boson production. If the Higgs boson is supersymmetric and if $\tan \beta$ is large, the diffractive production of MSSM Higgs bosons could lead to a discovery in the double pomeron exchange mode at the LHC. Figure 2 demonstrates that the signalover-background can reach a value up to $54,26,16$, and 13 for respective Higgs boson mass resolutions in roman pot detectors of $1,2,3$ and $4 \mathrm{GeV}$ and for a value of $\tan \beta$ of 50 for a luminosity of $100 \mathrm{fb}^{-1}$.

\section{PRODUCTION OF TOP (BOTTOM) SQUARK PAIRS}

\section{A. Theoretical framework}

In the MSSM, for each quark flavor $q$, there are two supersymmetric scalar partners $\tilde{q}_{L}$ and $\tilde{q}_{R}$ associated with the two fermion chiralities $q_{L}$ and $q_{R}$. In general, these scalars are not mass eigenstates; due to the presence of soft-supersymmetry-breaking terms which mix them, the $A$-terms $A_{q} y_{q} \tilde{Q}_{L} \tilde{q}_{R}^{\star} H+$ h.c., where $y_{q}$ is the Yukawa coupling of the quark $q$ and $H$ is one of the two MSSM Higgs boson doublet. The mass eigenstates $\tilde{q}_{1}$ and $\tilde{q}_{2}$ are obtained by diagonalizing the following $2 \times 2$ matrix [51]:

$$
\left(\begin{array}{cc}
M_{Q}^{2}+m_{q}^{2}+D_{L} & m_{q} X_{q} \\
m_{q} X_{q} & M_{R}^{2}+m_{q}^{2}+D_{R}
\end{array}\right)
$$

where $\quad D_{L} \equiv\left(T_{q}^{3}-Q_{q} \sin ^{2} \theta_{W}\right) m_{Z}^{2} \cos 2 \beta, \quad D_{R} \equiv$ $Q_{q} \sin ^{2} \theta_{W} m_{Z}^{2} \cos 2 \beta, \quad X_{q} \equiv A_{q}-\mu \cot \beta \quad$ for up-type squarks and $X_{q} \equiv A_{q}-\mu \tan \beta$ for down-type squarks. The soft-supersymmetry-breaking squark masses $M_{Q}$ and $M_{R}$ are of the order of the supersymmetry-breaking scale $M_{\text {SUSY }}$, and phenomenological constraints require the $A$-term parameter $A_{q}$ to be at most a few times $M_{\text {SUSY }}$ [52]. Neglecting the terms proportional to $m_{Z}^{2}$ in the squark mass matrix, the mass eigenvalues and the mixing angle, which relates the weak interaction eigenstates $\tilde{q}_{L, R}$ to the mass eigenstates $\tilde{q}_{1,2}$, are given by the following expressions:

$$
\begin{aligned}
m_{\tilde{q}_{1,2}}^{2} & =\frac{1}{2}\left(M_{Q}^{2}+M_{R}^{2}+m_{q}^{2} \mp \sqrt{\left(M_{Q}^{2}-M_{R}^{2}\right)^{2}+4 m_{q}^{2} X_{q}^{2}}\right), \\
\tan \theta_{\tilde{q}} & =\frac{2 m_{q} X_{q}}{M_{Q}^{2}-M_{R}^{2}} .
\end{aligned}
$$

In practise, the mixing is significant only if $m_{q} X_{q} \sim$ $M_{\text {SUSY }}^{2}$, which can occur for the top squark and, at large $\tan \beta$, also for the bottom squark. In this case, one can have a strong hierarchy between the two mass eigenstates, $m_{\tilde{q}_{1}} \ll m_{\tilde{q}_{2}}$. The $95 \%$ C.L. experimental bounds on the lightest top squark and bottom squark masses are $m_{\tilde{t}_{1}}>$ $95.7 \mathrm{GeV}$ and $m_{\tilde{b}_{1}}>89 \mathrm{GeV}$, respectively, while the bound on the other squarks is $250 \mathrm{GeV}$ [53] (the latter bound also applies to $\tilde{b}_{1}$ if mixing effects are small in the bottom squark sector). Although these bounds were derived under specific assumptions and may therefore not hold in some regions of the MSSM parameter space, they are rather robust.

In our experimental study, we consider the following values for the lightest top squark mass: $174.3 \mathrm{GeV}$ (i.e., $m_{\tilde{t}_{1}}=m_{t}$ ), $210 \mathrm{GeV}$ and $393 \mathrm{GeV}$. As we will see, the resolution that can be obtained on the top squark mass crucially depends on its decay width, which in turn is a function of the top squark mass and of the other MSSM parameters. If the top squark is very light, it is likely to be the next-to-lightest supersymmetric particle, assuming the lightest supersymmetric particle (LSP) is the lightest neutralino $\tilde{\chi}_{1}^{0}$. Then all two-body decay channels occurring at the tree level are closed. If in addition the tree-body decay channels $\tilde{t}_{1} \rightarrow b W^{+} \tilde{\chi}_{1}^{0}$ and $\tilde{t}_{1} \rightarrow b H^{+} \tilde{\chi}_{1}^{0}$ are kinematically not accessible, the main decay mode is expected to be the loop-induced flavor-violating decay $\tilde{t}_{1} \rightarrow c \tilde{\chi}_{1}^{0} \quad$ [54]. The 
decay width of the lightest top squark is then given by [54]:

$$
\Gamma\left(\tilde{t}_{1} \rightarrow c \tilde{\chi}_{1}^{0}\right)=\frac{g^{2}}{16 \pi}\left|f_{L 1}^{c}\right|^{2}|\epsilon|^{2} m_{\tilde{t}_{1}}\left(1-\frac{m_{\tilde{\chi}_{1}^{0}}^{2}}{m_{\tilde{t}_{1}}^{2}}\right)^{2},
$$

where $\left|f_{L 1}^{c}\right| \leq 1$ is the $\tilde{c}_{L^{-}} c_{L^{-}} \tilde{\chi}_{1}^{0}$ coupling, and $\epsilon$ is a flavorviolating insertion. The authors of Ref. [54] estimated $|\epsilon| \sim(1-4) \times 10^{-4}$ in mSUGRA, yielding $\Gamma\left(\tilde{t}_{1} \rightarrow c \tilde{\chi}_{1}^{0}\right) \lesssim$ $(0.085-1.4) \times 10^{-9} m_{\tilde{t}_{1}}\left[1-\left(m_{\tilde{\chi}_{1}^{+}} / m_{\tilde{t}_{1}}\right)^{2}\right]^{2}$; but depending on the mSUGRA parameters $|\epsilon|$ could be either much smaller or larger, in particular, at large $\tan \beta$ where $|\epsilon|$ behaves like $\tan ^{2} \beta$. In nonminimal SUGRA models, $|\epsilon|$ could even be of order one. However, in the regions of the MSSM parameter space where $\Gamma\left(\tilde{t}_{1} \rightarrow c \tilde{\chi}_{1}^{0}\right)$ is suppressed, the four-body decay modes $\tilde{t}_{1} \rightarrow b \tilde{\chi}_{1}^{0} f \bar{f}$ are likely to be dominant $[55,56]$. For larger top squark masses, the threebody decay channels $\tilde{t}_{1} \rightarrow b W^{+} \tilde{\chi}_{1}^{0}$ and $\tilde{t}_{1} \rightarrow b H^{+} \tilde{\chi}_{1}^{0}$ $[57,58]$ (and, if the sleptons are lighter than the lightest top squark, $\tilde{t}_{1} \rightarrow b \nu_{l} \tilde{l}^{+}$and $\left.\tilde{t}_{1} \rightarrow b \tilde{\nu}_{l} l^{+}[54,58,59]\right)$ open up and tend to dominate. Finally, when $m_{\tilde{t}_{1}}>m_{b}+m_{\tilde{\chi}_{1}^{+}}$ and $m_{\tilde{t}_{1}}>m_{t}+m_{\tilde{\chi}_{1}^{0}}$, the two-body decays $\tilde{t}_{1} \rightarrow b \tilde{\chi}_{1}^{+}$and $\tilde{t}_{1} \rightarrow t \tilde{\chi}_{1}^{0}$ become kinematically accessible and dominate the lightest top squark decays ${ }^{8}$ [60]. The partial decay width of $\tilde{t}_{1} \rightarrow b \tilde{\chi}_{1}^{+}$is given by:

$$
\Gamma\left(\tilde{t}_{1} \rightarrow b \tilde{\chi}_{1}^{+}\right)=\frac{g^{2}}{16 \pi}\left(l_{11}^{2}+k_{11}^{2}\right) m_{\tilde{t}_{1}}\left(1-\frac{m_{\tilde{\chi}_{1}^{+}}^{2}}{m_{\tilde{t}_{1}}^{2}}\right)^{2},
$$

where $l_{11}$ and $k_{11}$ are chargino couplings. Since $\left|l_{11}\right|$, $\left|k_{11}\right| \lesssim 1, \quad \Gamma\left(\tilde{t}_{1} \rightarrow b \tilde{\chi}_{1}^{+}\right) \lesssim 0.0085 m_{\tilde{t}_{1}}\left[1-\left(m_{\tilde{\chi}_{1}^{+}} / m_{\tilde{t}_{1}}\right)^{2}\right]^{2}$. The decay $\tilde{t}_{1} \rightarrow t \tilde{\chi}_{1}^{0}$ has a larger phase space suppression.

For our experimental study, we do not consider any specific benchmark scenario, but simply assume $m_{\tilde{t}_{1}}<$ $m_{\tilde{\chi}_{1}^{+}}$for the cases $m_{\tilde{t}_{1}}=174$ and $210 \mathrm{GeV}$. Then, we conservatively take $\Gamma_{\tilde{t}_{1}}=100 \mathrm{MeV}$ for $m_{\tilde{t}_{1}}=174$ and $210 \mathrm{GeV}$, although the actual decay width could be much smaller. For $m_{\tilde{t}_{1}}=393 \mathrm{GeV}$, we assume that the two-body decay $\tilde{t}_{1} \rightarrow b \tilde{\chi}_{1}^{+}$is accessible, and we take the decay width computed for SPS 1a, $\Gamma_{\tilde{t}_{1}}=1.8 \mathrm{GeV}$ [29].

\section{B. Stop production cross section and missing mass distribution}

At hadron colliders, top squarks can be produced at lowest QCD order via quark-antiquark annihilation and gluon-gluon fusion. In the present study, we are interested in the $J_{z}=0$, color-singlet gluon-gluon fusion cross section; it reads, at the parton level [21]:

$$
\frac{d \sigma_{\mathrm{LO}}}{d t}\left(g g \rightarrow \tilde{t}_{i} \overline{\tilde{t}}_{i}\right)=\frac{4 \pi}{12} \frac{\alpha_{s}^{2}}{s^{2}} \frac{m_{\tilde{t}_{i}}^{4}}{E_{T}^{4}},
$$

\footnotetext{
${ }^{8}$ We do not consider the decays $\tilde{t}_{1} \rightarrow t \tilde{g}$ and $\tilde{t}_{1} \rightarrow \tilde{b}_{i} W^{+}$, $\tilde{b}_{i} H^{+}$, since the gluinos and the other squarks are generally heavier than the lightest top squark.
}

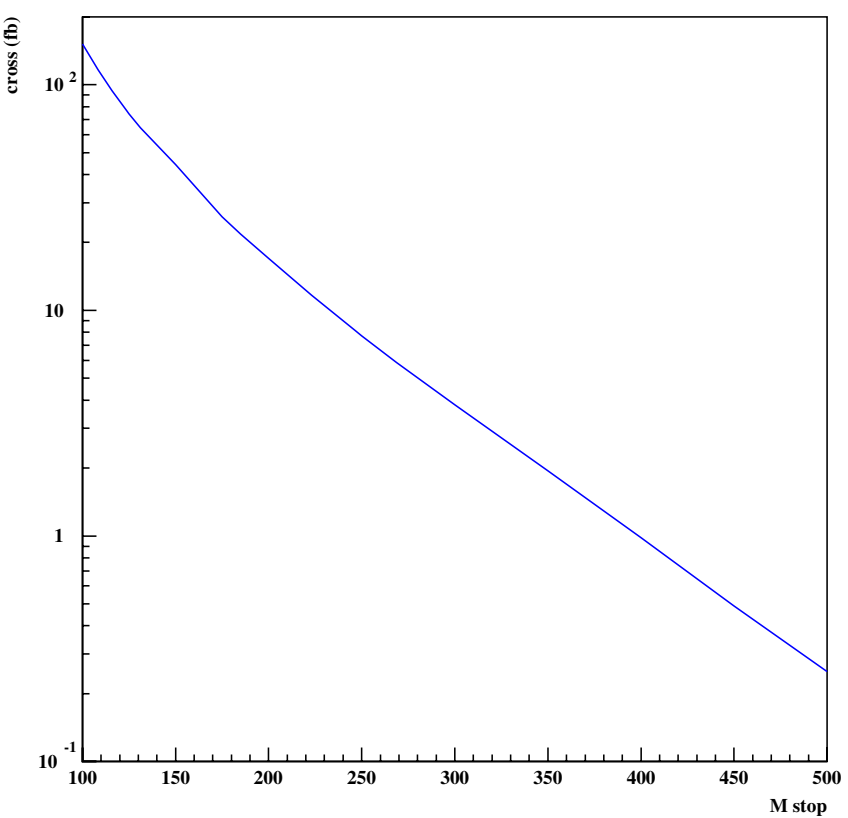

FIG. 3 (color online). Top squark pair production cross section as a function of the top squark mass.

where $\sqrt{s}$ is the center-of-mass energy of the subprocess, $m_{\tilde{t}_{i}}$ is the top squark mass, and $E_{T}$ is the transverse energy of the final particles.

The top squark production cross section has been obtained using the DPEMC generator [22] after applying a survival probability of 0.03 [61]. The top squark pair production cross section as a function of the top squark mass is given in Fig. 3. The $\tilde{t} \tilde{\bar{t}}$ production cross section is found to be 26.3, 14.1 and $1.1 \mathrm{fb}$ for a top squark mass of

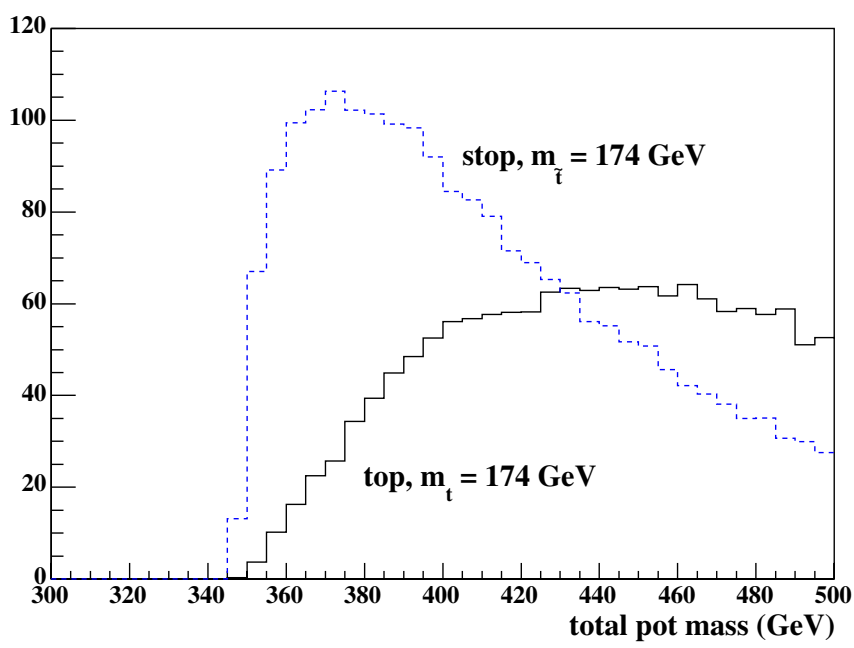

FIG. 4 (color online). Distribution of the missing mass for $100 \mathrm{fb}^{-1}$ for $t \bar{t}$ events (dashed line), and for $\tilde{t} \tilde{\bar{t}}$ (full line) for $m_{\text {top }}=m_{\tilde{t}}$. The faster rise of the stop quark cross section as a function of missing mass is due to the scalar nature of these particles. 


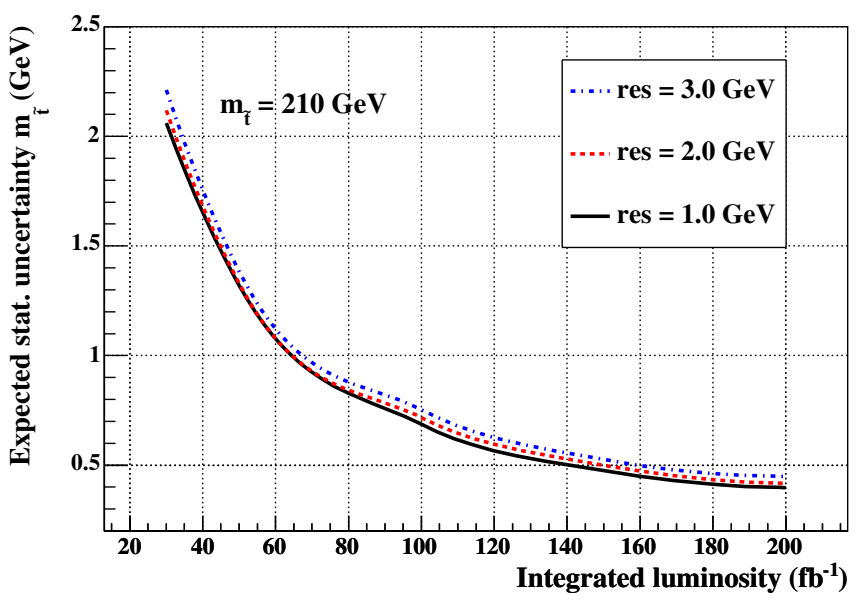

FIG. 5 (color online). Expected statistical precision of the $\tilde{t}$ mass as a function of the integrated luminosity for various resolutions of the roman pot detectors using the histogram-fitting method.

174.3 (at about the top quark mass), 210 and $393 \mathrm{GeV}$, respectively.

The distribution of the missing mass distribution for $t \bar{t}$ and $\tilde{t} \tilde{\bar{t}}$ events for $m_{\tilde{t}}=m_{t}=174.3 \mathrm{GeV}$ is shown in Fig. 4 for a luminosity of $100 \mathrm{fb}^{-1}$. The missing mass distribution for top squark (top) events is in full (dashed) line. The cross section rise at threshold is much faster than for top quarks and typical of pair production of scalar particles. The next section describes a method to determine the stop quark mass by performing a threshold scan of the missing mass of the $\tilde{t} \tilde{\bar{t}}$ process, measured with roman pot detectors.

\section{Stop mass measurement}

In this section, we describe briefly the method we used to obtain the stop mass resolution and its results. The histogram method ${ }^{9}$ is described in more detail in Ref. [62]. It compares the mass distribution in data with some reference distributions following a Monte Carlo simulation of the detector with different input masses corresponding to the data luminosity. As an example, we can produce a data sample for $100 \mathrm{fb}^{-1}$ with a top squark mass of $210 \mathrm{GeV}$, and a few MC samples corresponding to top squark masses between 180 and $240 \mathrm{GeV}$ by steps of $1 \mathrm{GeV}$. To evaluate the statistical uncertainty due to the method itself, we perform the fits with some 100 different "data" ensembles. For each ensemble, one obtains a different reconstructed top squark mass, the dispersion corresponding only to statistical effects. The $\chi^{2}$ is defined using the approximation of poissonian errors as given in Ref. [63]. Each ensemble thus gives a $\chi^{2}$ curve which in

\footnotetext{
${ }^{9}$ In Ref. [62], we give two methods to measure the $W$ boson or the top mass, namely, the histogram or the turn-on fit methods. For a matter of simplicity, we used only the histogram method in this paper.
}

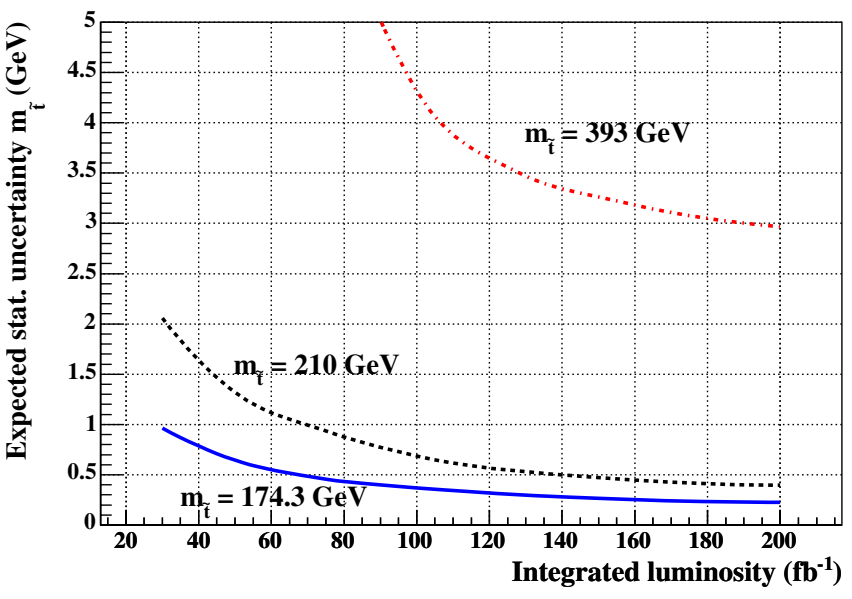

FIG. 6 (color online). Expected statistical precision of the $\tilde{t}$ mass as a function of the integrated luminosity for different $\tilde{t}$ masses (174.3, 210 and $393 \mathrm{GeV})$.

the region of the minimum is fitted with a fourth-order polynomial. The position of the minimum of the polynomial gives the best value of the top squark mass and the uncertainty $\sigma\left(m_{\tilde{t}}\right)$ is obtained from the values where $\chi^{2}=$ $\chi_{\min }^{2}+1$.

The results are given in Fig. 5 and 6. Figure 5 displays the results on the top squark mass resolution for a top squark mass of $210 \mathrm{GeV}$ as an example, as a function of luminosity, for different roman pot resolutions. The results depend only weakly on the roman pot resolution and mostly on the number of events produced for a given luminosity. The resolution on the top squark mass is thus dominated by statistics. We also note that the integrated luminosity does not take into account the efficiency of the cuts to select the $\tilde{t} \tilde{\bar{t}}$ events since these efficiencies depend strongly on the SUSY parameters. A typical efficiency of $60 \%$ is found requesting a missing transverse energy to be greater than $80 \mathrm{GeV}$, and either two reconstructed jets or one lepton and one jet with a transverse momentum greater than $20 \mathrm{GeV}$. Figure 6 displays the resolution obtained for the three values of the top squark mass discussed above. A resolution of about $0.4,0.7$ and $4.3 \mathrm{GeV}$ is obtained for a top squark mass of $174.3,210$ and $393 \mathrm{GeV}$ for a luminosity (divided by the signal efficiency) of $100 \mathrm{fb}^{-1}$. As it was mentioned in paragraph IVA, the top squark width has been taken into account in this study. For a top squark mass of $174.3,210 \mathrm{GeV}$, the top squark width of $100 \mathrm{MeV}$ has a negligible effect, whereas the top squark width of $1.8 \mathrm{GeV}$ for a top squark mass of $393 \mathrm{GeV}$ cannot be neglected.

\section{CONCLUSION AND OUTLOOK}

In this paper, we described the advantages of diffractive SUSY particle productions for two different processes, namely, the MSSM Higgs bosons and top squark pairs. The cross section for diffractive MSSM Higgs bosons 
production is noticeably enhanced at high values of $\tan \beta$ and since we look for Higgs boson decaying into $b \bar{b}$, it is possible to benefit directly from the enhancement of the cross section contrary to the nondiffractive case. A signalover-background up to a factor 50 can be reached for $100 \mathrm{fb}^{-1}$ for $\tan \beta \sim 50$. In particular, we analyzed in detail the antidecoupling regime, in which $H$ behaves like the SM Higgs boson, while $h$ has enhanced (resp. suppressed) couplings to down-type fermions (resp. up-type fermions and gauge bosons). We find that central diffraction production seems to be promising in that regime.

The other application is to use the so-called "thresholdscan method" to measure the top squark mass in exclusive events. The idea is quite simple: one measures the turn-on point in the missing mass distribution above twice the top squark mass. After taking into account the top squark width, we obtain a resolution on the top squark mass of $0.4,0.7$ and $4.3 \mathrm{GeV}$ for a top squark mass of $174.3,210$ and $393 \mathrm{GeV}$ for a luminosity (divided by the signal efficiency) of $100 \mathrm{fb}^{-1}$ and the production rates calculated in Sec. IV of this paper. If these rates hold, the typical mass resolutions are comparable to those at a linear collider. The process is thus similar to those at linear colliders (all final states are detected), but without the initial state radiation problem.

It should be stressed once more that production via the diffractive exclusive processes (1) is model dependent, and definitely needs the Tevatron data to test the models. It will allow to determine more precisely the production cross section by testing and measuring at the Tevatron the jet and photon production for high masses and high dijet or diphoton mass fraction. If, for instance, we compare (as much as possible since the methods of evaluation are not exactly comparable) the expectations of the Pomeroninduced [3,4] and proton-induced [7] models for exclusive production, we find striking differences for high mass states, in particular, the stop quark production. Indeed, the Sudakov form factors present in the proton-induced approach induce negligible stop quark cross sections [21]. The search for exclusive events in high mass dijet or diphoton events at the LHC is of considerable importance to test the models. For MSSM Higgs bosons in the lowmass range there is not an order-of-magnitude difference between the two models as was already mentioned for the standard Higgs production [16].

On the other hand, it is also possible to perform a similar study using inclusive double pomeron exchanges (2). These processes have already been observed by many experiments but suffer from the lack of knowledge on the gluon density in the pomeron at high $\beta$. The first step is thus to measure this gluon density by, for instance, using dijet events or the threshold-scan method for inclusive $t \bar{t}$ production. Once the high- $\beta$ gluon better determined, it is possible to look for top squark events, again using the threshold-scan method and deviation at high masses provided the cross section is high enough. This study goes beyond the purpose of the present paper and it certainly deserves a dedicated study [62].

\section{ACKNOWLEDGMENTS}

We thank Pavel Demine for his participation in this study at an early stage and for useful discussions.

\section{APPENDIX A: MSSM HIGGS BOSON PRODUCTION VIA GLUON FUSION AT LEADING ORDER}

The cross section for the lightest MSSM Higgs boson production via gluon fusion reads, at leading order [64]:

$$
\begin{aligned}
\sigma(g g \rightarrow h)= & \frac{G_{F} \alpha_{S}^{2}}{288 \sqrt{2} \pi} \mid \frac{3}{4} \sum_{q} g_{h q q} A_{q}^{h}\left(\tau_{q}\right) \\
& +\left.\frac{3}{4} \sum_{\tilde{q}} \frac{g_{h \tilde{q} \tilde{q}}}{m_{\tilde{q}}^{2}} A_{\tilde{q}}^{h}\left(\tau_{\tilde{q}}\right)\right|^{2},
\end{aligned}
$$

where the first term contains the quark loop contributions, and the second term the squark loop contributions. The loop functions $A_{q}^{h}(\tau)$ and $A_{\tilde{q}}^{h}(\tau)$ are given by:

$$
\begin{gathered}
A_{q}^{h}(\tau)=\frac{2}{\tau^{2}}[\tau+(\tau-1) f(\tau)], \\
A_{\tilde{q}}^{h}(\tau)=\frac{1}{\tau^{2}}[f(\tau)-\tau], \\
f(\tau)= \begin{cases}\arcsin ^{2}(\sqrt{\tau}) & \tau \leq 1 \\
-\frac{1}{4}\left[\ln \left(\frac{1+\sqrt{1-1 / \tau}}{1-\sqrt{1-1 / \tau}}\right)-i \pi\right]^{2} & \tau>1,\end{cases}
\end{gathered}
$$

and the parameters $\tau_{q}$ and $\tau_{\tilde{q}}$ are defined by $\tau_{q} \equiv m_{h}^{2} / 4 m_{q}^{2}$ and $\tau_{\tilde{q}} \equiv m_{h}^{2} / 4 m_{\tilde{q}}^{2}$, respectively, where $m_{q}$ (resp. $m_{\tilde{q}}$ ) denotes the mass of the quarks (resp. squarks) running in the loop. The couplings of the lightest MSSM Higgs boson to the top and bottom quarks, normalized to the SM Higgs boson couplings, are given by:

$$
\begin{gathered}
g_{h t t}=\sin (\beta-\alpha)+\cot \beta \cos (\beta-\alpha), \\
g_{h b b}=\sin (\beta-\alpha)-\tan \beta \cos (\beta-\alpha),
\end{gathered}
$$

and its couplings to the top squark and bottom squark mass eigenstates, in units of $g / M_{W}$, by (we omit the off-diagonal couplings $g_{h \tilde{t}_{1} \tilde{t}_{2}}$ and $g_{h \tilde{b}_{1} \tilde{b}_{2}}$, which are not relevant at leading order):

$$
g_{h \tilde{t}_{1} \tilde{t}_{1}}=-\left(\frac{1}{2} \cos ^{2} \theta_{\tilde{t}}-\frac{2}{3} \sin ^{2} \theta_{W} \cos 2 \theta_{\tilde{t}}\right) M_{Z}^{2} \sin (\beta+\alpha)+m_{t}^{2} \frac{\cos \alpha}{\sin \beta}+\frac{1}{2} \sin 2 \theta_{\tilde{t}} m_{t}\left(A_{t} \frac{\cos \alpha}{\sin \beta}+\mu \frac{\sin \alpha}{\sin \beta}\right)
$$




$$
\begin{aligned}
& g_{h \tilde{h}_{2} \tilde{t}_{2}}=-\left(\frac{1}{2} \sin ^{2} \theta_{\tilde{t}}+\frac{2}{3} \sin ^{2} \theta_{W} \cos 2 \theta_{\tilde{t}}\right) M_{Z}^{2} \sin (\beta+\alpha)+m_{t}^{2} \frac{\cos \alpha}{\sin \beta}-\frac{1}{2} \sin 2 \theta_{\tilde{t}} m_{t}\left(A_{t} \frac{\cos \alpha}{\sin \beta}+\mu \frac{\sin \alpha}{\sin \beta}\right), \\
& g_{h \tilde{b}_{1} \tilde{b}_{1}}=\left(\frac{1}{2} \cos ^{2} \theta_{\tilde{b}}-\frac{1}{3} \sin ^{2} \theta_{W} \cos 2 \theta_{\tilde{b}}\right) M_{Z}^{2} \sin (\beta+\alpha)-m_{b}^{2} \frac{\sin \alpha}{\cos \beta}+\frac{1}{2} \sin 2 \theta_{\tilde{b}} m_{b}\left(-A_{b} \frac{\sin \alpha}{\cos \beta}+\mu \frac{\cos \alpha}{\cos \beta}\right), \\
& g_{h \tilde{b}_{2} \tilde{b}_{2}}=\left(\frac{1}{2} \sin ^{2} \theta_{\tilde{b}}+\frac{1}{3} \sin ^{2} \theta_{W} \cos 2 \theta_{\tilde{b}}\right) M_{Z}^{2} \sin (\beta+\alpha)-m_{b}^{2} \frac{\sin \alpha}{\cos \beta}-\frac{1}{2} \sin 2 \theta_{\tilde{b}} m_{b}\left(-A_{b} \frac{\sin \alpha}{\cos \beta}+\mu \frac{\cos \alpha}{\cos \beta}\right),
\end{aligned}
$$

where $\theta_{\tilde{t}}$ and $\theta_{\tilde{b}}$ are the mixing angle in the stop and the bottom squark sector, respectively, defined by $\tilde{q}_{1}=\cos \theta_{\tilde{q}} \tilde{q}_{L}+$ $\sin \theta_{\tilde{q}} \tilde{q}_{R}, \tilde{q}_{2}=-\sin \theta_{\tilde{q}} \tilde{q}_{L}+\cos \theta_{\tilde{q}} \tilde{q}_{R}$.

The leading order cross section for the SM Higgs boson production via gluon fusion can be obtained from Eq. (A1) by removing the squark contribution and by setting $g_{h q q}=1$.

[1] A. Bialas and P. V. Landshoff, Phys. Lett. B 256, 540 (1991).

[2] A. Bialas and W. Szeremeta, Phys. Lett. B 296, 191 (1992); A. Bialas and R. Janik, Z. Phys. C 62, 487 (1994).

[3] M. Boonekamp, R. Peschanski, and C. Royon, Phys. Lett. B 598, 243 (2004).

[4] M. Boonekamp, R. Peschanski, and C. Royon, Phys. Rev. Lett. 87, 251806 (2001); M. Boonekamp, A. De Roeck, R. Peschanski, and C. Royon, Phys. Lett. B 550, 93 (2002); M. Boonekamp, R. Peschanski, and C. Royon, Nucl. Phys. B669, 277 (2003); B676, 493(E) (2004); for a general review see C. Royon, Mod. Phys. Lett. A 18, 2169 (2003).

[5] B. Cox, J. Forshaw, and B. Heinemann, Phys. Lett. B 540, 263 (2002).

[6] R. Enberg, G. Ingelman, A. Kissavos, and N. Timneanu, Phys. Rev. Lett. 89, 081801 (2002).

[7] V. A. Khoze, A. D. Martin, and M. G. Ryskin, Eur. Phys. J. C 19, 477 (2001); 20, 599(E) (2001); V. A. Khoze, A. D. Martin, and M. G. Ryskin, Eur. Phys. J. C 24, 581 (2002).

[8] J. D. Bjorken, Phys. Rev. D 47, 101 (1993); J. R. Cudell and O. F. Hernandez, Nucl. Phys. B471, 471 (1996); E. M. Levin, hep-ph/9912403 and references therein; J. Pumplin, Phys. Rev. D 52, 1477 (1995); A. Berera and J. C. Collins, Nucl. Phys. B474, 183 (1996).

[9] For recent reviews of supersymmetry and the MSSM, see e.g. S.P. Martin, hep-ph/9709356; D. J.H. Chung, L.L. Everett, G. L. Kane, S. F. King, J. Lykken, and L. T. Wang, Phys. Rep. 407, 1 (2005); I. J. R. Aitchison, hep-ph/ 0505105.

[10] B.E. Cox, J.R. Forshaw, J.S. Lee, J. Monk, and A. Pilaftsis, Phys. Rev. D 68, 075004 (2003).

[11] J.R. Ellis, J.S. Lee, and A. Pilaftsis, Phys. Rev. D 71, 075007 (2005).

[12] C. Royon, Mod. Phys. Lett. A 18, 2169 (2003).

[13] A. B. Kaidalov, V. A. Khoze, A.D. Martin, and M.G. Ryskin, Eur. Phys. J. C 33, 261 (2004).

[14] G. Albrow and A. Rostovtsev, hep-ph/0009336.

[15] D. Goulianos, Workshop on Diffraction at the LHC, Rio de Janeiro, 2004; M. Gallinaro (CDF - Run II Collaboration), hep-ph/0505159.
[16] For a recent discussion, see J.R. Forshaw, hep-ph/ 0508274. The phenomenological normalization factor 3.8 (see the introduction) has not been applied in the paper.

[17] A. Bzdak, Acta Phys. Pol. B 35, 1733 (2004); Phys. Lett. B 608, 64 (2005).

[18] J. D. Bjorken, Phys. Rev. D 47, 101 (1993); E. Gotsman, E. Levin, and U. Maor, Phys. Lett. B 438, 229 (1998); A. B. Kaidalov, V. A. Khoze, A.D. Martin, and M.G. Ryskin, Eur. Phys. J. C 21, 521 (2001); A. Bialas, Acta Phys. Pol. B 33, 2635 (2002); A. Bialas and R. Peschanski, Phys. Lett. B 575, 30 (2003).

[19] A. Kupco, C. Royon, and R. Peschanski, Phys. Lett. B 606, 139 (2005).

[20] A. Donnachie and P. V. Landshoff, Phys. Lett. B 207, 319 (1988).

[21] V. A. Khoze, A. D. Martin, and M. G. Ryskin, Eur. Phys. J. C 23, 311 (2002).

[22] M. Boonekamp and T. Kucs, Comput. Phys. Commun. 167, 217 (2005).

[23] H. E. Haber and Y. Nir, Phys. Lett. B 306, 327 (1993); H. E. Haber, hep-ph/9505240.

[24] J.F. Gunion and H.E. Haber, Phys. Rev. D 67, 075019 (2003).

[25] E. Boos, A. Djouadi, M. Muhlleitner, and A. Vologdin, Phys. Rev. D 66, 055004 (2002); E. Boos, A. Djouadi, and A. Nikitenko, Phys. Lett. B 578, 384 (2004).

[26] A. Djouadi, hep-ph/0503173.

[27] See e.g. J. F. Gunion, A. Stange, and S. Willenbrock, hepph/9602238.

[28] A. H. Chamseddine, R. Arnowitt, and P. Nath, Phys. Rev. Lett. 49, 970 (1982); R. Barbieri, S. Ferrara, and C. A. Savoy, Phys. Lett. 119B, 343 (1982); L. J. Hall, J. Lykken, and S. Weinberg, Phys. Rev. D 27, 2359 (1983).

[29] N. Ghodbane and H. U. Martyn, in Proceedings of the $A P S / D P F / D P B$ Summer Study on the Future of Particle Physics, edited by N. Graf (Snowmass, Colorado, 2001); N. Ghodbane and H. U. Martyn, hep-ph/0201233; B.C. Allanach et al., Eur. Phys. J. C 25, 113 (2002).

[30] N. Ghodbane, hep-ph/9909499.

[31] M. Battaglia, A. De Roeck, J. R. Ellis, F. Gianotti, K. A. Olive, and L. Pape, Eur. Phys. J. C 33, 273 (2004). 
[32] M. Drees, Phys. Rev. D 33, 1468 (1986); S. Dimopoulos and G. F. Giudice, Phys. Lett. B 357, 573 (1995); A. Pomarol and D. Tommasini, Nucl. Phys. B466, 3 (1996); E. Dudas, S. Pokorski, and C. A. Savoy, Phys. Lett. B 369, 255 (1996); A. G. Cohen, D. B. Kaplan, and A. E. Nelson, Phys. Lett. B 388, 588 (1996).

[33] N. Arkani-Hamed and H. Murayama, Phys. Rev. D 56, 6733 (1997).

[34] G. Corcella et al., J. High Energy Phys. 01 (2001) 010.

[35] J. Kalliopuska, T. Mäki, N. Marola, R. Orava, K. Österberg, and M. Ottela, HIP-2003-11/EXP.

[36] M. Carena and H. E. Haber, Prog. Part. Nucl. Phys. 50, 63 (2003).

[37] Y. Okada, M. Yamaguchi, and T. Yanagida, Prog. Theor. Phys. 85, 1 (1991).

[38] J. R. Ellis, G. Ridolfi, and F. Zwirner, Phys. Lett. B 257, 83 (1991).

[39] H. E. Haber and R. Hempfling, Phys. Rev. Lett. 66, 1815 (1991).

[40] H. E. Haber, R. Hempfling, and A. H. Hoang, Z. Phys. C 75, 539 (1997).

[41] J.F. Gunion and H.E. Haber, Nucl. Phys. B278, 449 (1986).

[42] A. Djouadi, M. Spira, and P. M. Zerwas, Phys. Lett. B 264, 440 (1991).

[43] S. Dawson, Nucl. Phys. B359, 283 (1991).

[44] M. Spira, A. Djouadi, D. Graudenz, and P. M. Zerwas, Nucl. Phys. B453, 17 (1995).

[45] S. Dawson, A. Djouadi, and M. Spira, Phys. Rev. Lett. 77, 16 (1996).

[46] R. V. Harlander and M. Steinhauser, Phys. Lett. B 574, 258 (2003).

[47] R. Harlander and M. Steinhauser, Phys. Rev. D 68, 111701 (2003).

[48] R. V. Harlander and M. Steinhauser, J. High Energy Phys. 09 (2004) 066.

[49] A. Djouadi, Phys. Lett. B 435, 101 (1998).

[50] CMS Collab., CMSIM, fast simulation of the CMS detec- tor, Technical Design Report 1997); TOTEM Collab., Technical Design Report, Report No. CERN/LHCC/997; ATLAS Collab., ATLFAST, fast simulation of the ATLAS detector, Technical Design Report, Report No. CERN/LHC C/99-14.

[51] J. R. Ellis and S. Rudaz, Phys. Lett. 128B, 248 (1983); M. Drees and K. I. Hikasa, Phys. Lett. B 252, 127 (1990).

[52] J. M. Frere, D. R. T. Jones, and S. Raby, Nucl. Phys. B222, 11 (1983); H. P. Nilles, M. Srednicki, and D. Wyler, Phys. Lett. 120B, 346 (1983); J. P. Derendinger and C. A. Savoy, Nucl. Phys. B237, 307 (1984).

[53] S. Eidelman et al. (Particle Data Group), Phys. Lett. B 592, 1 (2004).

[54] K. I. Hikasa and M. Kobayashi, Phys. Rev. D 36, 724 (1987).

[55] C. Boehm, A. Djouadi, and Y. Mambrini, Phys. Rev. D 61, 095006 (2000).

[56] A. Djouadi, M. Guchait, and Y. Mambrini, Phys. Rev. D 64, 095014 (2001).

[57] W. Porod and T. Wohrmann, Phys. Rev. D 55, 2907 (1997); 67, 059902(E) (2003).

[58] W. Porod, Phys. Rev. D 59, 095009 (1999).

[59] A. Datta, M. Guchait, and K. K. Jeong, Int. J. Mod. Phys. A 14, 2239 (1999).

[60] For reviews of the two-body decays of the lightest top squark, see e.g. W. Porod, hep-ph/9804208; S. Kraml, hep$\mathrm{ph} / 9903257$.

[61] V. A. Khoze, A. D. Martin, and M. G. Ryskin, Eur. Phys. J. C 18, 167 (2000).

[62] M. Boonekamp, J. Cammin, R. Peschanski, and C. Royon, hep-ph/0504199.

[63] N. Gehrels, Astrophys. J. 303, 336 (1986).

[64] J. F. Gunion, H. E. Haber, G. L. Kane, and S. Dawson, The Higgs Hunter's Guide (Addison-Wesley, Reading, 1990).

[65] S. Heinemeyer, W. Hollik, and G. Weiglein, Comput. Phys. Commun. 124, 76 (2000); M. Frank, S. Heinemeyer, W. Hollik, and G. Weiglein, hep-ph/0202166. 